\title{
ESTADO DE LA COMUNICACIÓN DE RESPONSABILIDAD SOCIAL CORPORATIVA EN EL SECTOR HOTELERO ESPAÑOL
}

\author{
Andrea Pérez \\ Ignacio Rodríguez del Bosque \\ Universidad de Cantabria
}

\section{RESUMEN}

El objetivo de los autores de este trabajo es analizar la comunicación de responsabilidad social corporativa (RSC) de las empresas hoteleras en España mediante la aplicación de un marco teórico que integra las perspectivas del desarrollo sostenible y de los grupos de interés. Los resultados muestran que la información de RSC ofrecida en este sector se encuentra actualmente dispersa entre los informes y páginas web de las empresas analizadas. Por lo tanto, los autores identifican varias áreas de mejora en la gestión y comunicación de RSC en el sector hotelero.

Palabras clave: Responsabilidad social corporativa; desarrollo sostenible; grupos de interés; hoteles; comunicación.

\section{Corporate social responsibility communication in the spanish hospitality sector}

\section{ABSTRACT}

The aim of the authors in this paper is to analyse the online corporate social responsibility (CSR) communication of hospitality companies in Spain by applying a theoretical framework that integrates two major perspectives used in the study of CSR in the tourism industry: sustainable development and stakeholder theories. The findings show that the CSR information communicated in this sector is currently scattered through the

Recibido: 12 de marzo de 2014

Devuelto para su revisión: 25 de septiembre de 2014

Aceptado: 22 de octubre de 2014

Área de Comercialización e Investigación de Mercados. Facultad de Ciencias Económicas y Empresariales. Universidad de Cantabria. Avenida Los Castros, s/n. 39005 SANTANDER (España). E-mail: perezran@unican. es,rbosquei@unican.es 
reports and websites of the companies analysed. Thus, the authors identify several areas for improvement in the CSR management and communication in the hospitality sector.

Keywords: Corporate social responsibility; sustainable development; stakeholders; hotels; communication.

\section{INTRODUCCIÓN}

El turismo es una de las principales industrias de servicios en todo el mundo. En términos generales genera cerca de 2.000 millones de euros anuales y da empleo a un $15 \%$ de la población activa mundial. Por lo tanto, si se utiliza de manera responsable, el turismo puede ser una fuerza para el crecimiento sostenible y el éxito económico de muchos países (Dodds y Kuehnel, 2010). Sin embargo, si se utiliza de forma irresponsable, también puede ser una fuente de conflicto pues supondrá salarios bajos, empleo estacional, inestabilidad y pobres condiciones laborales, degradación medioambiental, desplazamiento de la población local, inflación y deterioro de recursos culturales (Agarwal, 2002). En este contexto, al sector turístico se le reclaman nuevas estrategias y formas de demostrar sus principios éticos y de respecto al bienestar social y al crecimiento sostenible de la economía. Por ello, la Responsabilidad Social Corporativa (RSC) ha cobrado un especial impulso en el sector turístico, donde las empresas deben desempeñar un papel fundamental en el ejercicio de prácticas de buen gobierno (Martínez et al., 2013).

En su búsqueda de nuevos caminos para ayudar a los profesionales a cumplir con las exigencias impuestas por el nuevo contexto económico y social, los investigadores han centrado recientemente su atención en el estudio de los beneficios de la denominada comunicación de RSC. La comunicación de RSC se refiere a la divulgación de información por parte de las empresas de aquellas actividades llevadas a cabo y que demuestran la inclusión de compromisos económicos, sociales y medioambientales en las operaciones comerciales de las empresas así como en las relaciones con los grupos de interés. Los investigadores han argumentado teóricamente que la divulgación de información de RSC es parte del diálogo entre una empresa ética y sus grupos de interés que ayuda a legitimar el comportamiento corporativo y, como tal, contribuye a generar una mejor reputación corporativa (Michelon, 2011).

En el contexto específico del sector hotelero, el estudio de la comunicación de RSC es muy importante porque los grupos de interés han manifestado que les gustaría tener información sistemática sobre las prácticas socialmente responsables de este tipo de empresas tan influyentes en la economía mundial. Sin embargo, los mismos grupos de interés han señalado también que, por lo general, suele ser muy difícil obtener la información necesaria para satisfacer sus necesidades porque los informes de RSC son confusos y la forma de comunicar la información no es homogénea entre las empresas (Holcomb et al., 2007). Junto a esta limitación cabe destacar que, en este contexto específico, aún parece existir insuficiente investigación académica sobre la comunicación de RSC de las empresas hoteleras (Jones et al., 2006; Holcomb et al., 2007; de Grosbois, 2012; Font et al., 2012).

A partir de estas ideas, el objetivo de los autores en el presente trabajo es analizar la comunicación de RSC que llevan a cabo a través de Internet las empresas del sector 
hotelero en España. Con ello, se busca también identificar las fallas que presenta dicha comunicación de RSC para poder proponer sugerencias de mejora de cara a la subsanación de las mismas (Font et al., 2012).

En este sentido, en el ámbito de la RSC la comunicación online es una de las herramientas más potentes de mejora de la imagen de una empresa (Jones et al, 2006; Holcomb et al., 2007), ya que se ha demostrado que los grupos de interés prefieren que este tipo de prácticas de RSC se comuniquen a través de los llamados canales 'minoritarios' (como los informes anuales y/o páginas web) frente al uso de los canales de masas tradicionales (Morsing y Schultz, 2006). Para el estudio, los autores realizan un análisis de contenidos de la información pública de RSC ofrecida por una muestra de 170 empresas del sector, incluyendo grandes cadenas hoteleras así como pequeños y medianos hoteles de gestión independiente.

Dicho análisis se realiza desde una perspectiva que combina las ideas planteadas tanto por la teoría del desarrollo sostenible como por la teoría de los grupos de interés. Éstas son dos de las principales perspectivas conceptuales que se han utilizado para el estudio de la RSC en el sector hotelero. Sin embargo, en estudios previos se ha evidenciado que los investigadores y gestores de la comunicación de RSC en las empresas muy pocas veces integran el conocimiento generado por ambas corrientes de opinión (Jones et al., 2006; Holcomb et al., 2007; Martínez et al., 2013), de tal manera que los avances realizados hasta la fecha son parciales y no permiten tener una visión global de la RSC en el sector hotelero. La integración de ambas perspectivas en el modelo global de comunicación planteado en este trabajo representa otra de las contribuciones significativas del artículo.

Tal y como se mostrará en los siguientes epígrafes, los principales resultados y conclusiones del trabajo confirman que las empresas del sector hotelero español utilizan las páginas web corporativas principalmente como herramientas comerciales, hecho que se pone de manifiesto en la cantidad de información ofrecida a los clientes, que son el grupo de interés mejor atendido a través de los medios electrónicos. A diferencia de otros sectores de actividad, la información facilitada a través de las webs corporativas también incluye referencias significativas al patrimonio natural y los recursos culturales de los destinos turísticos, como es lógico, ya que estos son aspectos que afectan directamente a la capacidad para atraer turistas de los destinos y empresas hoteleras. Así, por tanto, los resultados de este trabajo reflejan claramente el carácter contextual de la RSC en España, demostrando que las implicaciones de este concepto en el sector turístico son muy diferentes a las implicaciones en otros contextos económicos.

Con el ánimo de cumplir con los objetivos de la investigación, el presente artículo se estructura de la siguiente manera. En primer lugar, se realiza una breve revisión de los conceptos de RSC y comunicación empresarial en el sector hotelero, que permiten proponer dos cuestiones de estudio. Las mismas se testan a través de un estudio de contenidos cuya metodología se presenta en la cuarta sección del trabajo. Finalmente, se presentan los principales resultados de la investigación así como sus principales conclusiones, limitaciones y futuras líneas de investigación.

\section{LA RSC EN EL SECTOR HOTELERO}

La RSC en el sector hotelero se ha definido tradicionalmente a partir de dos perspectivas teóricas. En primer lugar, la mayoría de los investigadores consideran que la teoría 
del desarrollo sostenible ofrece el enfoque más adecuado para el estudio de la RSC en este contexto (Martínez et al., 2013). De acuerdo con esta perspectiva, la RSC se entiende como un constructo multidimensional que enfatiza igualmente las funciones económicas, sociales y medioambientales de las empresas. Estas tres dimensiones conforman la denominada 'triple cuenta de resultados' de la empresa.

La dimensión económica se basa en asegurar actividades económicas viables a largo plazo, de manera que todos los interesados reciban beneficios socioeconómicos distribuidos equitativamente. La dimensión social se refiere a un respeto por la autenticidad de los valores tradicionales de los destinos turísticos, así como a la contribución de las empresas a la comprensión y tolerancia intercultural. La dimensión medioambiental se refiere a la utilización óptima del medioambiente, que es un elemento esencial del desarrollo del turismo. De esta forma, incluye aspectos como la protección de los procesos ecológicos esenciales y la ayuda de las empresas a la conservación de los recursos naturales y la biodiversidad de los destinos turísticos.

No obstante, en este trabajo los autores proponen añadir una cuarta dimensión, vinculada con aspectos culturales, al estudio del desarrollo sostenible en el sector hotelero. En este sentido, el sector hotelero es muy sensible a las cuestiones culturales (Bohdanowicz y Zientara, 2008) y, por lo tanto, los autores consideran que las prácticas culturales deben constituir una dimensión con identidad propia en el estudio de la RSC. Concretamente, muchos hoteles están situados en núcleos urbanos, a menudo situados cerca de representaciones significativas del patrimonio cultural de los mismos, que atraen a un creciente número de viajeros y, consecuentemente, imponen así un mayor impacto sobre los recursos culturales (de Grosbois, 2012). Igualmente importante resulta el fenómeno de las relaciones sociales entre los turistas y los residentes locales, que es considerado como una importante fuerza impulsora del respeto y el entendimiento entre naciones y culturas. Por lo tanto, el turismo puede ayudar positivamente a propagar los valores culturales que yacen en el corazón de los destinos turísticos (Bohdanowicz y Zientara, 2008) y, de esta forma, su inclusión en el estudio de la RSC es esencial.

En segundo lugar, los investigadores también han analizado la RSC desde el punto de vista de la teoría de los grupos de interés (Freeman, 1984). En este sentido, se considera que la comunicación de las acciones pro-sociales (económicas, sociales o medioambientales) puede contribuir muy poco a la mejora de la reputación de una empresa si la sociedad percibe que ésta es simultáneamente dañina para sus grupos de interés, o incluso que está intentando engañar a la opinión pública sobre este tipo de asuntos (Esrock y Leichty, 1998). Por lo tanto, la teoría de los grupos de interés defiende que la RSC debe ser evaluada sobre la base de los grupos de interés que más se benefician de las iniciativas prosociales, ya que dichos colectivos son los destinatarios de cada conducta corporativa. A este respecto, y siguiendo las propuestas de autores previos como Jones et al. (2006), los autores de este trabajo proponen analizar la comunicación de RSC realizada a seis grupos de interés, considerados los más relevantes en el ámbito de la gestión hotelera: clientes (turistas), proveedores, empleados, accionistas e inversores, sociedad y medioambiente.

Así, por tanto, el estudio de la comunicación de RSC en el sector hotelero debe realizarse teniendo en cuenta las dos perspectivas teóricas planteadas, tal y como muestra la Figura 1. 


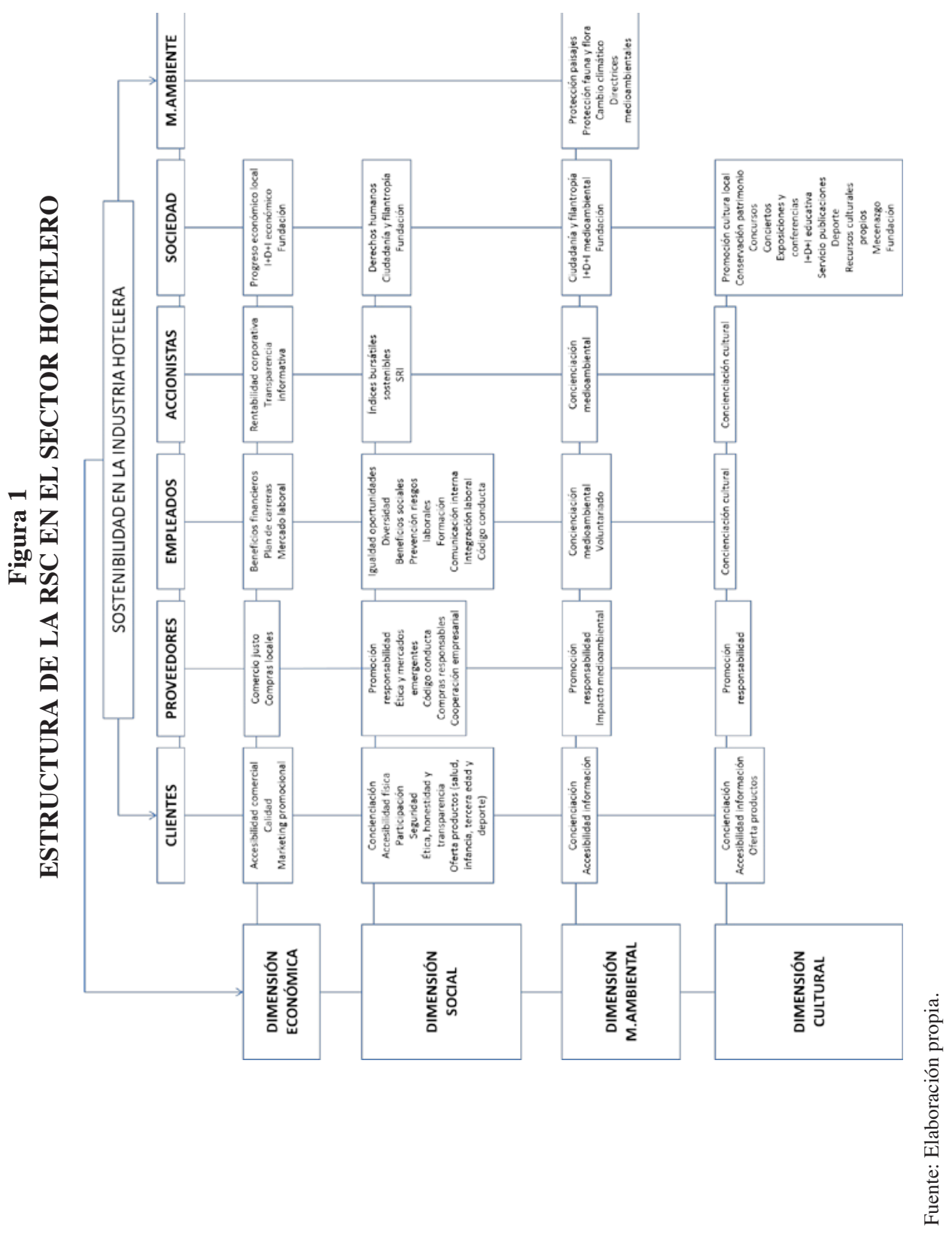




\section{LA COMUNICACIÓN DE RSC COMO HERRAMIENTA DE GENERACIÓN DE REPUTACIÓN CORPORATIVA}

Las empresas están cada vez más interesadas en informar sobre sus actividades de RSC (Font et al., 2012), ya que los grupos de interés cada vez quieren saber más acerca de las organizaciones que se encuentran detrás de las marcas y los productos que compran y, como tal, buscan una mayor transparencia y rendición de cuentas por parte de las mismas (de Grosbois, 2012). En este contexto, se ha demostrado que la comunicación de RSC presenta una muy buena oportunidad de dar forma a la imagen de marca y las creencias públicas sobre la empresa (Pomering y Dolnicar, 2009).

Además, dicha transmisión de información puede producirse a través de múltiples canales de comunicación, como los informes y memorias de RSC, los medios de comunicación masiva o Internet (de Grosbois, 2012). Sin embargo, y debido a la naturaleza sensible de la comunicación de RSC, los investigadores han llegado a la conclusión de que este tipo de prácticas no pueden ser objeto del mismo tipo de comunicación que se utiliza para productos, servicios o marcas. En esta línea, la confianza en la fuente de información es fundamental para el éxito de la comunicación de RSC. Por ejemplo, los grupos de interés, especialmente los consumidores, tienen una tendencia natural a ser escépticos con la publicidad, más que con otras fuentes de información. De esta forma, comunicar la RSC a través de publicidad tradicional se percibe como una actividad subjetiva, más bien enfocada a acentuar las buenas acciones de la empresa, lo que puede conducir a reticencias hacia el mensaje y cinismo sobre los verdaderos motivos altruistas de la empresa que realiza prácticas de RSC y las comunica a través de este medio. Este hecho, por tanto, plantea un desafío mucho más complejo para los especialistas de la comunicación de RSC.

En esta misma línea, recientemente se ha demostrado que la comunicación de RSC a través de medios online es mucho más efectiva para construir una buena imagen de marca que cualquier otro tipo de canal de comunicación (Esrock y Leichty, 1998). En este sentido, las páginas web tienen varias características atractivas cuando se trata de la auto-presentación de una empresa. En primer lugar, las páginas web sirven a audiencias que son más activas en la forma en que buscan y procesan la información. También se pueden diseñar mensajes que no sufren tanto de las restricciones temporales que presentan los medios de comunicación masiva, como la prensa escrita o electrónica. En tercer lugar, las páginas web también pueden emplear características interactivas para recopilar información, hacer seguimiento de la opinión pública y conseguir que los ciudadanos participen activamente en el diálogo directo sobre una variedad de asuntos económicos, sociales, medioambientales y culturales (Grösch, 2011).

Esta importancia de la comunicación online para transmitir información de RSC ha llevado a diversos investigadores a interesarse por el estudio de la misma en el contexto hotelero (Holcomb et al., 2007; de Grosbois, 2012; Font et al., 2012). Sin embargo, los estudios son aún pocos y, además, están centrados en todos los casos en contextos diferentes al español. Por tanto, de las ideas planteadas a lo largo de este artículo se desprende la necesidad de ahondar en dos cuestiones de investigación de especial relevancia para la gestión de la comunicación de RSC en el sector hotelero: 
- ¿Qué tipo de comunicación de RSC se está desarrollando en el sector hotelero español?

- ¿Qué sugerencias y recomendaciones se derivan del estudio de dicha comunicación?

\section{METODOLOGÍA}

Para responder a estas cuestiones de investigación, los autores realizaron un análisis de contenidos de las páginas web de 170 empresas hoteleras. En concreto, la muestra incluía tanto cadenas hoteleras como hoteles independientes, seleccionados a través de una técnica de muestreo probabilístico y estratificado (con asignación proporcional, aleatoria y sistemática). La selección de las empresas se realizó partiendo de una base de datos de 2.862 empresas hoteleras con páginas web disponibles y activas, que los autores crearon a partir de la información proporcionada por Hostelmarket (revista mensual de información hostelera española). La Tabla 1 resume el perfil de la investigación.

Tabla 1

PERFIL DE LA INVESTIGACIÓN

\begin{tabular}{ll}
\hline Universo & 2.862 hoteles con página web activa \\
\hline Alcance & España \\
\hline Fecha trabajo de campo & Enero 2012 - Marzo 2012 \\
\hline Muestra & $\begin{array}{l}170 \text { empresas hoteleras } \\
(41 \text { cadenas y } 129 \text { hoteles })\end{array}$ \\
\hline Procedimiento de muestreo & $\begin{array}{l}\text { Aleatorio estratificado con asignación proporcional }(\mathrm{y} \\
\text { elección aleatoria sistemática })\end{array}$ \\
\hline Error muestral & $7,79 \%$ para un nivel de confianza del $95 \%(\mathrm{p}=\mathrm{q}=0,5)$ \\
\hline \hline
\end{tabular}

Fuente: Elaboración propia.

Para realizar el análisis de contenido los autores siguieron el procedimiento sugerido por Bravo et al. (2012). De esta manera, se utilizaron dos jueces independientes para explorar los diferentes tipos de información divulgada en los sitios web de las empresas hoteleras, incluyendo informes anuales (financieros, de gestión y de RSC) y toda la información que se mostraba directamente en los enlaces internos de las páginas web (Bravo et al., 2012). Este proceso permitió a los autores abordar una limitación de los estudios anteriores, que se centraban exclusivamente en el análisis de los informes de RSC sin tener en cuenta otros enlaces web que también contienen información adicional de RSC (Bravo et al., 2012).

Los jueces independientes eran profesores de marketing contratados en la misma universidad de los autores de este trabajo. Siguiendo las ideas de Bakeman y Gottman (1986) y Bravo et al. (2012), se estableció un sistema de supervisión y capacitación para estos jueces. Con este fin, y después de que los autores habían explicado los objetivos de la investigación a los jueces, re realizó un primer examen independiente de algu- 
nos de los sitios web corporativos, tanto por parte de los jueces como de los autores. Posteriormente, se celebró una reunión programada con el fin de discutir los incidentes más relevantes y obtener consenso en los criterios de análisis. Se establecieron también reuniones regulares para discutir nuevos incidentes y refinar todo el proceso de recogida de información.

Tras el análisis, se calcularon los coeficientes de acuerdo «entre jueces» para cada una de las categorías analizadas. El propósito de esta estimación era conseguir un coeficiente de concordancia suficiente para asegurar que el estudio empírico era suficientemente riguroso. Los coeficientes de concordancia en las categorías analizadas fueron en todos los casos superiores al $90 \%$, aceptable para este tipo de método (Neüendorf, 2002) y similar a los alcanzados en estudios previos (Gram, 2007). Además, el horizonte temporal del análisis abarcaba tres meses (enero 2012-marzo 2012), de modo que la variabilidad de la información contenida en las páginas web, resultado del dinamismo de la comunicación en este canal, se recogiese correctamente (Bravo et al., 2012).

El primer paso en el análisis de contenido consistió en la definición de un sistema de clasificación de las diferentes dimensiones de la comunicación de RSC. La clasificación inicial fue creada por los propios autores, aunque se diseñó de manera flexible para dar cabida también a la nueva información recopilada por los jueces independientes (Bravo et al., 2012). Para garantizar la validez del procedimiento, como punto de partida se consideraron las cuatro categorías de desarrollo sostenible y las seis de gestión de grupos de interés presentadas anteriormente en este trabajo (Bravo et al., 2012). Por lo tanto, los autores definieron una dimensión económica, social, ambiental y cultural para cada una de las seis dimensiones de grupos de interés identificadas en este artículo (clientes, proveedores, empleados, accionistas, sociedad y medio ambiente).

Los autores comenzaron definiendo una clasificación teórica de 59 ítems de RSC, que se basaban en las propuestas de estudios anteriores que habían analizado la comunicación de RSC de empresas en diversas industrias (Clarkson, 1995; Ayuso y Fullana, 2002; Perrini et al., 2006; Holcomb et al., 2007; Merwe y Wocke, 2007; Bravo et al., 2012; Williams y Adams, 2013). Por ejemplo, en el dominio de los clientes, las categorías económicas se refieren a temas de accesibilidad comercial, calidad de productos y servicios y marketing promocional (Perrini et al, 2006; Bravo et al., 2012). Las categorías sociales se refieren a aspectos de concienciación social y promoción de la RSC entre los clientes, accesibilidad física, participación en asuntos sociales, seguridad, ética, honestidad y transparencia y oferta comercial de productos saludables para grupos de interés específicos (niños o ancianos, entre otros) (Clarkson, 1995; Perrini et al, 2006; Merwe y Wocke, 2007; Bravo et al., 2012). Las categorías ambientales incluyen la conciencia ambiental y la accesibilidad a la información ambiental (Merwe y Wocke, 2007). Por último, las categorías culturales también incluyen la conciencia cultural y la accesibilidad a la información (Merwe y Wocke, 2007).

En lo que se refiere a la dimensión de los proveedores, los autores definieron una categoría económica en relación a la promoción de condiciones justas de comercio. La dimensión social incluye cinco categorías: promoción de la RSC, ética en mercados emergentes, códigos de conducta, compras responsables y cooperación empresarial (Bravo et al., 2012). La dimensión ambiental incluye una categoría que se refiere a la promoción 
de esta dimensión de RSC también entre los proveedores. En esta etapa no se propusieron categorías culturales, ya que estudios previos no habían considerado esta perspectiva.

En el dominio de los empleados, las categorías económicas incluyen beneficios financieros, planificación de la carrera profesional y actividades orientadas al mercado de trabajo (Clarkson, 1995; Ayuso y Fullana, 2002; Holcomb et al., 2007; Merwe y Wocke, 2007; Bravo et al., 2012). Las categorías sociales están relacionadas con la igualdad de oportunidades, la diversidad, las prestaciones sociales, la prevención de riesgos laborales, la formación, la comunicación interna, la integración laboral y los códigos internos de conducta (Clarkson, 1995; Exceltur, 2003; Perrini et al., 2006; Holcomb et al., 2007; Bravo et al., 2012). Las categorías ambientales incluyen la conciencia ambiental y el voluntariado (Holcomb et al., 2007). Por último, tampoco se propusieron categorías culturales iniciales en esta dimensión de empleados.

En lo que se refiere a los accionistas, sólo se propusieron dos dimensiones de desarrollo sostenible en esta etapa. En este caso, se propusieron categorías económicas relacionadas con el suministro de información sobre la rentabilidad empresarial y la transparencia (Clarkson, 1995; Holcomb et al., 2007). Por otro lado, se propusieron también dos categorías sociales relativas a la inclusión de la empresa en índices de RSC y a la inversión socialmente responsable (Exceltur, 2003).

La dimensión de la sociedad incluye tres categorías económicas (concretamente sobre el progreso económico local, el presupuesto destinado a I+D y la creación de una fundación dedicada a estos temas) (Exceltur, 2003; Holcomb et al, 2007; Bravo et al., 2012); tres categorías sociales (en materia de derechos humanos, ciudadanía y filantropía y, nuevamente, la creación de una fundación) (Clarkson, 1995; Exceltur, 2003); tres categorías ambientales (ciudadanía y filantropía, presupuesto de I+D y fundación) (Exceltur, 2003; Bravo et al., 2012); y, finalmente, diez categorías culturales (promoción de la cultura local, conservación del patrimonio, concursos, conciertos, exposiciones y conferencias, presupuesto de $\mathrm{I}+\mathrm{D}$, servicio de publicaciones, deportes, recursos culturales propios y fundación) (Clarkson, 1995; Ayuso y Fullana, 2002; Exceltur, 2003; Merwe y Wocke, 2007; Bravo et al., 2012).

El grupo de interés denominado medio ambiente incluye tres categorías de información ambiental. Estas categorías se refieren a la protección de la flora y la fauna, el cambio climático y las pautas ambientales de la organización (Clarkson, 1995; Merwe y Wocke, 2007; Bravo et al., 2012).

Dentro de cada categoría, los autores también identificaron diferentes ítems con el fin de no perder información útil (Bravo et al., 2012). A modo de ejemplo, la categoría de calidad de productos y servicios (dimensión económica orientada a los clientes) contiene cinco códigos llamados «política de calidad», «certificación UNE-EN-ISO 9001:2008», «certificación Q», «certificación TripAdvisor»y «otras certificaciones». La categoría referida a la participación de los clientes en temas sociales (dimensión social orientada a los clientes) incluye tres códigos llamados «encuestas de satisfacción», «buzón de sugerencias» y «participación de los clientes en actividades socialmente responsables». Sin embargo, durante la recogida de información llevada a cabo por los jueces independientes, éstos identificaron nuevas categorías e ítems que fueron añadidos a la clasificación inicial con el fin de lograr una clasificación más precisa 
(Florek et al., 2006; Rowley, 2009). La aparición de estas nuevas categorías e ítems requirió que los jueces revisasen todos los sitios web de nuevo, para emplear el mismo esquema de categorización para todos los sitios web y obtener así información coherente y comparable (Bravo et al., 2012).

Finalmente se analizaron 67 categorías de información de RSC. Las siete nuevas categorías de información fueron: (1) la oferta comercial de productos culturales para clientes; (2) la medición del impacto ambiental de las empresas proveedoras; (3) la promoción de la RSC cultural entre los proveedores; (4) la generación de concienciación medioambiental entre los proveedores; (5) la concienciación cultural de los accionistas; (6) el patrocinio de actividades culturales (sociedad) y (7) la comunicación de información relativa a la protección paisajística promovida por la empresa. Estas siete categorías están en su mayoría relacionadas con las dimensiones cultural y medioambiental de la teoría del desarrollo sostenible, las dos dimensiones menos exploradas en la literatura previa.

En total se utilizaron 123 ítems, clasificados en las 67 categorías de RSC definidas para el estudio. La información se distribuye de acuerdo a las siguientes proporciones: 34 códigos en la dimensión del cliente ( 7 son códigos económicos; 19 sociales; 3 medioambientales y 5 culturales); 12 códigos en la dimensión de proveedores ( 1 es un código social; 8 sociales; 2 medioambientales y 1 cultural); 21 códigos en la dimensión de empleados (4 son códigos económicos; 15 sociales; 1 medioambiental y 1 cultural); 10 códigos en la dimensión de accionistas (6 son códigos económicos; 2 sociales; 1 medioambiental y 1 cultural) y 31 códigos en la dimensión de la sociedad (5 son códigos económicos; 5 sociales; 4 medioambientales y 17 culturales). Por último, 15 códigos se incluyen en la dimensión medioambiental (todos los códigos referidos a la dimensión medioambiental de la teoría del desarrollo sostenible). La Tabla 2 muestra un resumen de todas las categorías de información analizadas en este trabajo, junto a los trabajos en los que está basada la clasificación final.

Durante este proceso, la información de RSC se midió de manera similar a la base de datos KLD (Kinder, Lydenberg y Domini), que comprende evaluaciones numéricas en siete áreas de RSC: comunidad, gobierno corporativo, diversidad de la fuerza de trabajo, relaciones laborales, medio ambiente, derechos humanos y producto. En este sentido, a cada empresa hotelera se le dio un valor de 0 o 1 en cada ítem de RSC dependiendo de si la empresa comunicaba sobre el mismo (1) o no (0). La valoración de los códigos respondió a la frase «Esta empresa informa sobre las iniciativas llevadas a cabo (o los resultados logrados) en este campo concreto de la RSC». La puntuación final de una empresa en una categoría de RSC era la media ponderada de las evaluaciones que había recibido en cada uno de los ítems que forman la categoría.

Por último, es necesario precisar que, a los efectos de este estudio, los autores se centran en el porcentaje de las empresas hoteleras que informan sobre cada una de las categorías y dimensiones del enfoque de RSC considerado en este trabajo. 
Tabla 2

DIMENSIONES Y CATEGORÍAS DEL ANÁLISIS DE CONTENIDOS DE LA COMUNICACIÓN DE RSC

\begin{tabular}{|c|c|c|c|}
\hline $\begin{array}{l}\text { Dimensiones } \\
\text { desarrollo } \\
\text { sostenible }\end{array}$ & $\begin{array}{l}\text { Grupos de } \\
\text { interés }\end{array}$ & Categorías de información de RSC & Referencias \\
\hline \multirow{14}{*}{ Clientes } & \multirow{3}{*}{ Económica } & 1. Accesibilidad comercial* & \multirow{3}{*}{ Perrini et al. (2006); Bravo et al. (2012) } \\
\hline & & 2. Calidad* & \\
\hline & & 3. Marketing promocional* & \\
\hline & \multirow{6}{*}{ Social } & 4. Concienciación* & \multirow{6}{*}{$\begin{array}{l}\text { Clarkson (1995); Perrini et al. (2006); } \\
\text { Merwe y Wöcke (2007); Bravo et al. } \\
\text { (2012) }\end{array}$} \\
\hline & & 5. Accesibilidad física* & \\
\hline & & 6. Participación del cliente* & \\
\hline & & 7. Acciones de seguridad* & \\
\hline & & 8. Ética, honestidad y transparencia* & \\
\hline & & $\begin{array}{l}\text { 9. Oferta de productos (salud, infancia, tercera edad, } \\
\text { deporte)* }\end{array}$ & \\
\hline & \multirow{2}{*}{ Medioambiental } & 10. Concienciación* & \multirow{2}{*}{ Merwe and Wöcke (2007) } \\
\hline & & 11. Accesibilidad información* & \\
\hline & \multirow{3}{*}{ Cultural } & 12. Concienciación* & \multirow{3}{*}{ Merwe y Wöcke (2007) } \\
\hline & & 13. Accesibilidad información* & \\
\hline & & 14. Oferta de productos & \\
\hline \multirow{9}{*}{ Proveedores } & Económica & 15. Comercio justo* & Merwe y Wöcke (2007) \\
\hline & \multirow{5}{*}{ Social } & 16. Concienciación* & \multirow{5}{*}{ Bravo et al. (2012) } \\
\hline & & 17. Ética y mercados emergentes* & \\
\hline & & 18. Código de conducta* & \\
\hline & & 19. Compras responsables* & \\
\hline & & 20. Cooperación empresarial* & \\
\hline & \multirow{2}{*}{ Medioambiental } & 21. Concienciación* & \multirow{2}{*}{ Bravo et al. (2012) } \\
\hline & & 22. Impacto en cadena de suministro & \\
\hline & Cultural & 23. Concienciación & Nueva categoría \\
\hline \multirow{14}{*}{ Empleados } & \multirow{3}{*}{ Económica } & 24. Beneficios financieros* & \multirow{3}{*}{$\begin{array}{l}\text { Clarkson (1995); Ayuso y Fullana } \\
\text { (2002); Merwe y Wöcke (2007); } \\
\text { Holcomb et al. (2007); Bravo et al. } \\
\text { (2012) }\end{array}$} \\
\hline & & 25. Plan de carreras* & \\
\hline & & 26. Mercado laboral* & \\
\hline & \multirow{8}{*}{ Social } & 27. Igualdad de oportunidades* & \multirow{8}{*}{$\begin{array}{l}\text { Clarkson (1995); Exceltur (2003); } \\
\text { Perrini et al. (2006); Holcomb et al. } \\
\text { (2007); Bravo et al. (2012) }\end{array}$} \\
\hline & & 28. Diversidad* & \\
\hline & & 29. Beneficios sociales* & \\
\hline & & 30. Prevención de riesgos laborales* & \\
\hline & & 31. Formación* & \\
\hline & & 32. Herramientas de Comunicación interna* & \\
\hline & & 33. Integración laboral* & \\
\hline & & 34. Código de conducta* & \\
\hline & \multirow{2}{*}{ Medioambiental } & 35. Concienciación* & \multirow{2}{*}{$\begin{array}{l}\text { Holcomb et al. (2007); Bravo et al. } \\
(2012)\end{array}$} \\
\hline & & 36. Voluntariado* & \\
\hline & Cultural & 37. Concienciación & Nueva categoría \\
\hline
\end{tabular}




\begin{tabular}{|c|c|c|c|}
\hline \multirow{6}{*}{ Accionistas } & \multirow{2}{*}{ Económica } & 38. Rentabilidad corporativa* & \multirow{2}{*}{ - Clarkson (1995); Holcomb et al. (2007) } \\
\hline & & 39. Transparencia informativa* & \\
\hline & \multirow{2}{*}{ Social } & 40. Inclusión en índices bursátiles sostenibles* & \multirow{2}{*}{ Exceltur (2003) } \\
\hline & & 41. Inversión Socialmente Responsable (SRI)* & \\
\hline & Medioambiental & 42. Concienciación & Nueva categoría \\
\hline & Cultural & 43. Concienciación & Nueva categoría \\
\hline \multirow{20}{*}{ Sociedad } & \multirow{3}{*}{ Económica } & 44. Progreso económico local* & \multirow{3}{*}{$\begin{array}{l}\text { Exceltur (2003); Holcomb et al. (2007) } \\
\text { - Bravo et al. (2012) }\end{array}$} \\
\hline & & 45. I+D económico* & \\
\hline & & 46. Fundación* & \\
\hline & \multirow{3}{*}{ Social } & 47. Derechos humanos* & \multirow{3}{*}{ Clarkson (1995); Exceltur (2003) } \\
\hline & & 48. Ciudadanía y filantropía* & \\
\hline & & 49. Fundación* & \\
\hline & \multirow{3}{*}{ Medioambiental } & 50. Ciudadanía y filantropía* & \multirow{3}{*}{ Exceltur (2003); Bravo et al. (2012) } \\
\hline & & 51. I+D medioambiental* & \\
\hline & & 52. Fundación* & \\
\hline & \multirow{11}{*}{ Cultural } & 53. Promoción cultura local* & \multirow{11}{*}{$\begin{array}{l}\text { Clarkson (1995); Ayuso y Fullana } \\
\text { (2002); Exceltur (2003); Merwe y } \\
\text { Wöcke (2007); Bravo et al. (2012) }\end{array}$} \\
\hline & & 54. Conservación del patrimonio* & \\
\hline & & 55. Concursos* & \\
\hline & & 56. Conciertos* & \\
\hline & & 57. Exposiciones y conferencias* & \\
\hline & & 58. I+D educativa* & \\
\hline & & 59. Servicio de publicaciones* & \\
\hline & & 60. Deporte* & \\
\hline & & 61. Recursos culturales propios* & \\
\hline & & 62. Mecenazgo & \\
\hline & & 63. Fundación* & \\
\hline \multirow{4}{*}{ M.ambiente } & \multirow{4}{*}{ Medioambiental } & 64. Protección de paisajes & \multirow{4}{*}{$\begin{array}{l}\text { Clarkson (1995); Merwe y Wöcke } \\
\text { (2007); Bravo et al. (2012) }\end{array}$} \\
\hline & & 65. Protección flora y fauna* & \\
\hline & & 66. Cambio climático* & \\
\hline & & 67. Directrices medioambientales* & \\
\hline
\end{tabular}

Nota: las categorías marcadas con un * hacen referencia a las 59 categorías de comunicación de RSC propuestas originalmente por los autores. El resto de categorías son nuevas, en el sentido de que fueron identificadas por los jueces independientes una vez comenzado el análisis de contenidos de las páginas web.

\section{RESULTADOS}

Un primer paso para entender la comunicación online de RSC en el sector hotelero consiste en saber cuántas empresas están trabajando en cualquier aspecto relacionado con las diversas dimensiones que este concepto comprende. La Tabla 3 contiene una breve descripción del tipo de comunicación que se está produciendo en este sector.

Según los resultados, se observa que aún pocas empresas utilizan los canales de comunicación online para transmitir información de RSC, un hecho particularmente evidente en los hoteles de gestión independiente que conforman la muestra de este estudio. En concreto, sólo el 10\% de las empresas hoteleras que operan en España tienen una sección específica dedicada a la RSC en sus páginas web, un porcentaje que se eleva al $34 \%$ entre las cadenas hoteleras pero que queda limitado al $2 \%$ entre los hoteles independientes. 
Tabla 3

HERRAMIENTAS DE COMUNICACIÓN DE RSC

\begin{tabular}{lccc}
\hline Herramientas de comunicación & $\begin{array}{c}\text { \% total de } \\
\text { empresas }\end{array}$ & $\begin{array}{c}\text { \% de } \\
\text { cadenas }\end{array}$ & $\begin{array}{c}\text { \% de hoteles } \\
\text { independientes }\end{array}$ \\
\hline Portal web de RSC & 10 & 34 & 2 \\
\hline Informe o memoria de RSC & 5 & 20 & 1 \\
\hline Sección de prensa & 19 & 41 & 12 \\
\hline Portales web específicos: & & & \\
1. Accionistas e inversores & 4 & 15 & 1 \\
2. Empleados y mercado laboral & 21 & 59 & 9 \\
3. Proveedores & 1 & 2 & - \\
4. Intermediarios & 18 & 51 & 8 \\
\hline Redireccionamiento a otros organismos & 24 & 29 & 22 \\
\hline Medios de comunicación específicos & 18 & 27 & 16 \\
\hline
\end{tabular}

Fuente: Elaboración propia.

Por otra parte, estos porcentajes se reducen drásticamente cuando se analiza la publicación de memorias de RSC, con sólo el $20 \%$ de las cadenas hoteleras y el $1 \%$ de los hoteles independientes publicando regularmente tales documentos. Hay que tener también en cuenta que la publicación de informes de RSC es una práctica relativamente reciente en el sector, ya que los primeros informes datan de finales de los años 2000 (2007 y 2008 principalmente). Además, la comunicación de RSC está aún poco estructurada en el sector hotelero y la información aparece dispersa a lo largo de las distintas secciones que componen los portales web corporativos de las empresas.

Por otra parte, el $98 \%$ de las cadenas hoteleras y el $76 \%$ de los hoteles independientes de la muestra utilizan motores de reserva online en sus sitios web. Esto apunta a un carácter fuertemente comercial del canal de comunicación online, más allá del papel de generación de reputación corporativa que se le ha atribuido tradicionalmente.

También se observa que en el sector hotelero existen diferencias significativas en la relación comunicativa que las empresas establecen con diferentes grupos de interés, así como en el volumen de empresas que reportan información sobre las diversas dimensiones del desarrollo sostenible definidas teóricamente en este estudio.

Por un lado, la Figura 2 y la Tabla 4 muestran los porcentajes de empresas que comunican diversos aspectos relacionados con la RSC hacia sus grupos de interés. Los autores realizan una comparación de medias con el programa estadístico SPSS v.19, utilizando el estadístico t de Student y un nivel de significación del 95\%, para evaluar si existen diferencias sustanciales en el número de empresas que comunican información en cada categoría de grupos de interés. Concretamente, la interpretación del estadístico t señala que si este valor es superior a 1.96 y lleva asociado un p-valor (probabilidad) inferior a 0.05 , las diferencias evaluadas son estadísticamente significativas. En caso contrario (t-student $<1.96$ y p-valor $>0.05$ ), las diferencias entre categorías no son estadísticamente significativas y no puede concluirse que el volumen de empresas sea sustancialmente diferente entre categorías de información. 
Siguiendo este razonamiento, si se analizan los resultados mostrados en la Tabla 4 se observa que las diferencias existentes entre la gran mayoría de categorías estudiadas son los suficientemente importantes como para concluir que la comunicación de RSC en Internet se dirige especialmente a determinados grupos de interés, tales como los clientes, si bien es una herramienta apenas aplicada para gestionar la relación con otros colectivos, como los accionistas o proveedores.

En concreto, se observa que el mayor volumen de empresas reportan información dirigida a clientes $(94,7 \%$ de todas las empresas de la muestra). Un porcentaje significativamente menor de las empresas también incluye información orientada a la sociedad $(36,5 \%)$, empleados $(26,5 \%)$, medioambiente $(22,9 \%)$, proveedores $(9,4 \%)$ y accionistas e inversores $(6,5 \%)$. Por tanto, estos datos confirman el carácter comercial otorgado a los sitios web de las empresas hoteleras, que utilizan esta herramienta como un canal esencial para la comunicación con los clientes actuales y potenciales.

Además, se observan también diferencias notables en los volúmenes de empresas que comunican la información en función de que se clasifiquen como cadenas hoteleras o como hoteles independientes. El segundo tipo de empresas comunican información de RSC en mucha menor medida, algo que coincide plenamente con resultados previos hallados en la literatura académica y que señalan que las empresas de tamaño medio y/o pequeño generalmente son menos activas en términos de comunicación que las grandes empresas.

\section{Figura 2 \\ COMUNICACIÓN A LOS GRUPOS DE INTERÉS}

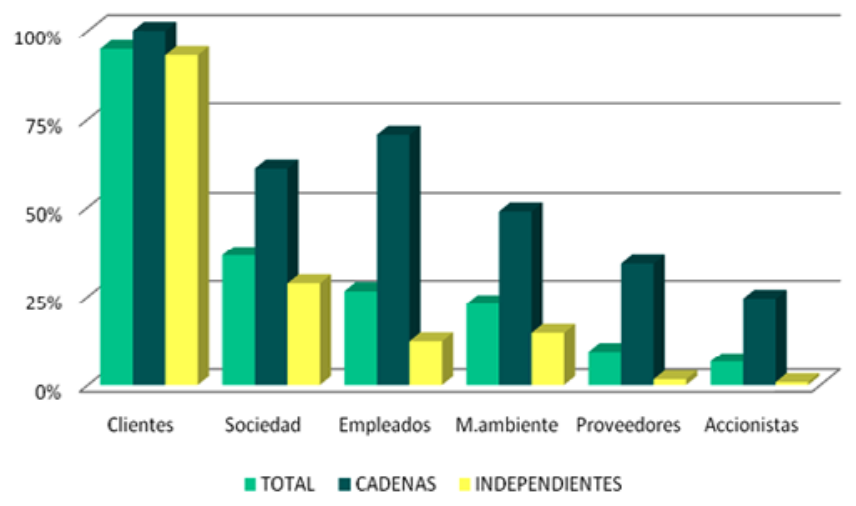

Fuente: Elaboración propia.

Por otra parte, si este mismo análisis de comparación de medias se realiza para la comunicación de las cuatro dimensiones del desarrollo sostenible definidas en este trabajo, se observa que también existen diferencias sustanciales entre los volúmenes de hoteles que aportan información en cada una de estas categorías (Tabla 5). En concreto, el análisis de contenidos muestra que un elevado número de empresas proporcionan información social (91,8 \% de todas las empresas de la muestra), seguido de información medioambiental $(76,5 \%)$, cultural $(74,7 \%)$ y, finalmente, información económica $(38,2 \%)$. 
Tabla 4

\section{COMPARACIÓN DE MEDIAS DE LA COMUNICACIÓN A LOS GRUPOS DE INTERÉS}

\begin{tabular}{|c|c|c|c|c|c|}
\hline \multirow[b]{2}{*}{ Grupos de interés } & \multirow{2}{*}{$\begin{array}{l}\% \text { total de } \\
\text { empresas }\end{array}$} & \multicolumn{2}{|c|}{ Comparación de medias } & \multirow[b]{2}{*}{ DT } & \multirow{2}{*}{$\begin{array}{l}\text { Significación } \\
\text { T-test (p-valor) }\end{array}$} \\
\hline & & $\begin{array}{l}\text { Grupos de } \\
\text { interés }\end{array}$ & Media & & \\
\hline \multirow{5}{*}{ Clientes } & \multirow{5}{*}{94,7} & Proveedores & 0,85 & 0,36 & $37,06(0,00)$ \\
\hline & & Empleados & 0,68 & 0,47 & $23,08(0,00)$ \\
\hline & & Accionistas & 0,88 & 0,32 & $39,03(0,00)$ \\
\hline & & Sociedad & 0,58 & 0,51 & $14,71(0,00)$ \\
\hline & & M.ambiente & 0,72 & 0,46 & $20,57(0,00)$ \\
\hline \multirow{4}{*}{ Proveedores } & \multirow{4}{*}{9,4} & Empleados & 0,17 & 0,38 & $3,95(0,00)$ \\
\hline & & Accionistas & 0,03 & 0,20 & $0,58(0,06)^{*}$ \\
\hline & & Sociedad & 0,27 & 0,47 & $6,90(0,00)$ \\
\hline & & M.ambiente & 0,14 & 0,34 & $4,41(0,00)$ \\
\hline \multirow{3}{*}{ Empleados } & \multirow{3}{*}{26,5} & Accionistas & 0,20 & 0,42 & $3,83(0,00)$ \\
\hline & & Sociedad & 0,10 & 0,51 & $3,98(0,01)$ \\
\hline & & M.ambiente & 0,04 & 0,45 & $0,65(0,31)^{*}$ \\
\hline \multirow{2}{*}{ Accionistas } & \multirow{2}{*}{6,5} & Sociedad & 0,30 & 0,47 & $6,78(0,00)$ \\
\hline & & M.ambiente & 0,17 & 0,37 & $4,56(0,00)$ \\
\hline Sociedad & 36,5 & M.ambiente & 0,14 & 0,51 & $3,20(0,00)$ \\
\hline M.ambiente & 22,9 & - & - & - & - \\
\hline
\end{tabular}

La conclusión más relevante derivada de este análisis se refiere a la integración de la dimensión cultural en el concepto de comunicación de RSC en el sector hotelero. En este caso, si bien la teoría académica considera que los aspectos culturales no constituyen en sí una dimensión del desarrollo sostenible, el análisis empírico en el sector hotelero muestra que este pilar tiene autoridad suficiente para ser considerado una dimensión en sí misma. Esta dimensión es aún más importante en la comunicación de RSC del sector hotelero que el componente económico.

Los resultados presentados también pueden desglosarse por categorías de información, tal y como se mostró en la Figura 1 de este trabajo. En este sentido, cabe destacar que la estructura propuesta a nivel teórico en este trabajo para estudiar la comunicación de RSC en el sector hotelero es casi totalmente representativa de la comunicación efectuada por las principales cadenas hoteleras y hoteles independientes que operan en España. Sin embargo, en ambas muestras se detectan algunas lagunas que cabe destacar.

Por ejemplo, ni las cadenas hoteleras ni los hoteles independientes proporcionan información económica a los proveedores. Tampoco proporcionan información medioambiental ni cultural a empleados o accionistas. 


\section{Figura 3 \\ COMUNICACIÓN DE LAS DIMENSIONES DE DESARROLLO SOSTENIBLE}

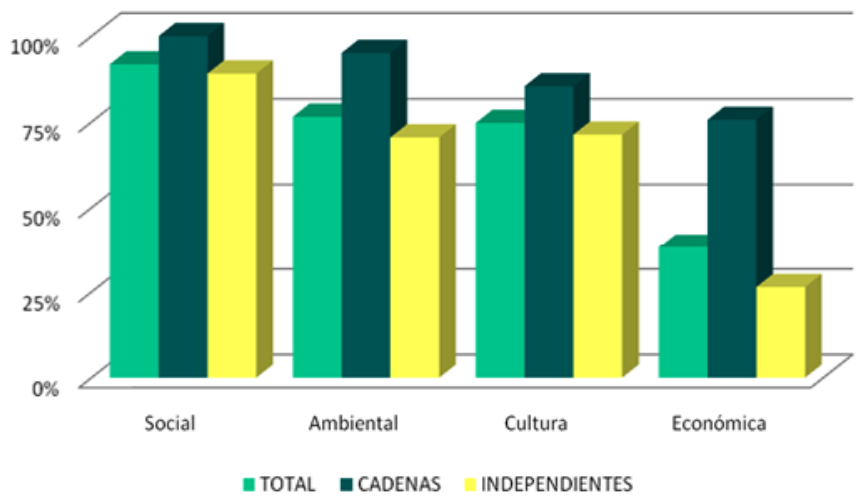

Fuente: Elaboración propia.

Tabla 5

COMPARACIÓN DE MEDIAS DE LA COMUNICACIÓN

DE LAS DIMENSIONES DE DESARROLLO SOSTENIBLE

\begin{tabular}{|c|c|c|c|c|c|}
\hline \multirow{2}{*}{ Dimensiones } & \multirow{2}{*}{$\begin{array}{l}\% \text { total de } \\
\text { empresas }\end{array}$} & \multicolumn{3}{|c|}{ Comparación de medias } & \multirow{2}{*}{$\begin{array}{l}\text { Significación } \\
\text { T-test (p-valor) }\end{array}$} \\
\hline & & Dimensiones & Media & DT & \\
\hline \multirow{3}{*}{ Económica } & \multirow{3}{*}{38,2} & Social & 0,54 & 0,54 & $13,42(0,00)$ \\
\hline & & Medioambiental & 0,38 & 0,54 & $8,78(0,00)$ \\
\hline & & Cultural & 0,37 & 0,57 & $8,92(0,00)$ \\
\hline \multirow{2}{*}{ Social } & \multirow{2}{*}{91,8} & Medioambiental & 0,15 & 0,44 & $4,40(0,00)$ \\
\hline & & Cultural & 0,17 & 0,46 & $4,12(0,00)$ \\
\hline Medioambiental & 76,5 & Cultural & 0,18 & 0,52 & $0,16(0,66)^{*}$ \\
\hline Cultural & 74,7 & - & - & - & - \\
\hline
\end{tabular}

Por otra parte, en la Figura 5 se puede observar que la dimensión social de la relación con los accionistas y la dimensión cultural de la relación con los proveedores no son parte de la comunicación de RSC llevada a cabo por los hoteles independientes. También es notable que un cierto número adicional de categorías de información tampoco formen parte de la comunicación de RSC de los hoteles independientes, un hecho que diferencia de manera significativa a estas empresas de las cadenas hoteleras que operan en España. En concreto, estas categorías se refieren a (1) la transparencia informativa con accionistas, (2) la igualdad de oportunidades, la diversidad, la prevención de riesgos y la integración laboral en el área de empleados, (3) la promoción de la responsabilidad 

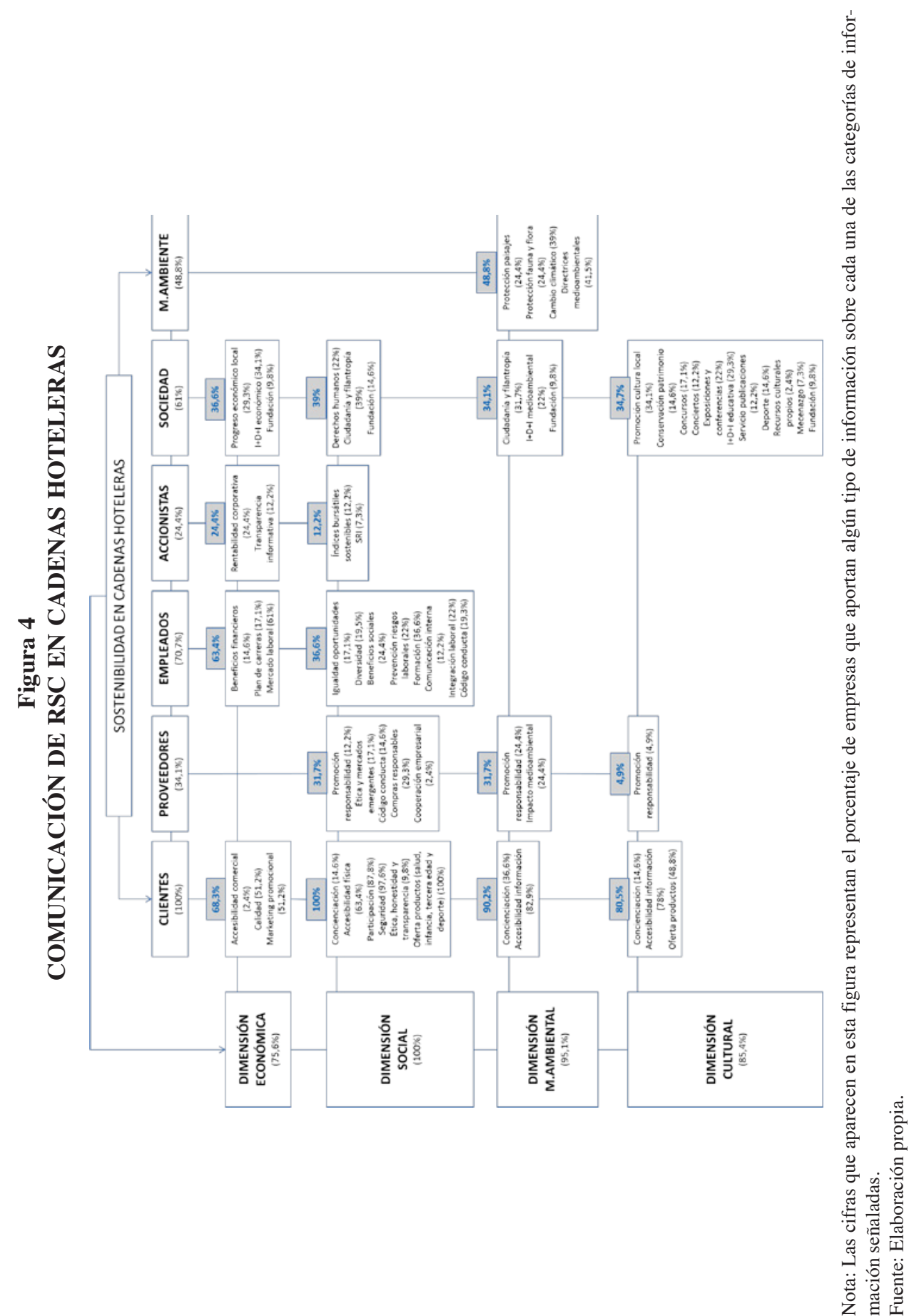


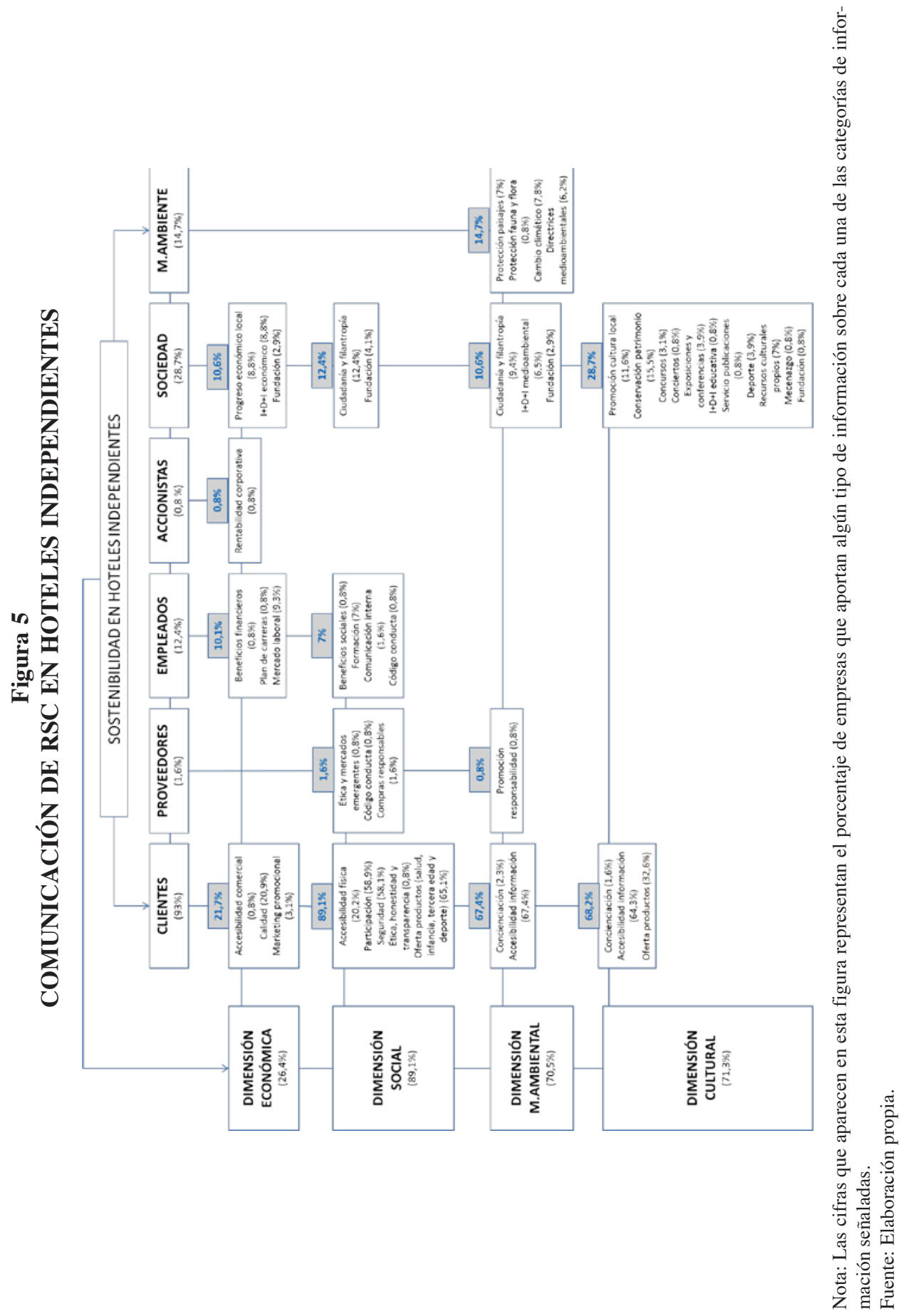


social, la cooperación empresarial y la estimación de la impacto medioambiental en el ámbito de los proveedores, (4) la concienciación social de los clientes y (5) la protección de los derechos humanos con respecto a la sociedad. Estos resultados no vienen sino a confirmar el bajo perfil comunicativo que tienen los hoteles independientes en materia de RSC.

En cuanto a la cantidad de empresas que reportan información en cada categoría, los resultados destacan la importancia de la dimensión social orientada a clientes, tanto entre las cadenas hoteleras ( $100 \%$ de las empresas informan sobre este tema) como entre los hoteles independientes $(89,1 \%)$. Las dimensiones medioambiental $(90,2 \%$ y $67,4 \%$, respectivamente) y cultural $(80,5 \%$ y $68,2 \%$, respectivamente) también destacan.

Las principales diferencias entre los dos tipos de empresas en este campo están en el tratamiento que se da a los empleados: mientras que son el segundo grupo de interés por volumen de cadenas hoteleras que lo incluyen en su comunicación de RSC, en el caso de los hoteles independientes son más las empresas que priorizan el aporte de información a la sociedad y el medioambiente antes que a los empleados.

\section{CONCLUSIONES FINALES}

El objetivo de los autores en este trabajo ha sido el de analizar la comunicación de RSC que llevan a cabo a través de Internet las empresas del sector hotelero en España, a fin de determinar qué dimensiones del concepto (económica, social, medioambiental y cultural) se reportan en mayor medida y cuáles son los grupos de interés (clientes, proveedores, empleados, accionistas, sociedad y medio ambiente) que más información reciben por parte tanto de cadenas hoteleras como de hoteles de gestión independiente. A fin de explorar estos aspectos, en el artículo se han presentado los principales resultados de un análisis de contenidos de 170 páginas web de empresas hoteleras para las que primero se ha elaborado un índice de comunicación al que posteriormente se han aplicado tests de comparación de medias.

La primera conclusión relevante de este trabajo hace referencia a la idoneidad mostrada por la estructuración del estudio de la comunicación de RSC a partir de la combinación de las ideas propuestas por las teorías del desarrollo sostenible y los grupos de interés. En el sector hotelero, dicha estructuración atiende al estudio de las dimensiones económica, social, medioambiental y cultural del desarrollo sostenible así como a la comunicación de RSC realizada a clientes (turistas), proveedores, empleados, accionistas e inversores, sociedad y medioambiente.

La validez de la propuesta se demuestra a partir de la aplicación de la misma al estudio de la comunicación tanto de grandes cadenas hoteleras como de medianos y pequeños hoteles de gestión independiente, dos tipos de empresas con perfiles comunicativos muy diferentes.

Esta nueva aproximación al estudio de la comunicación de RSC también permite a los autores completar las propuestas teóricas que se han utilizado hasta el momento en el mundo académico y profesional, por lo que proporciona una imagen más detallada de los niveles de desarrollo y puesta en práctica de la RSC en el sector hotelero. 
Los resultados también confirman el uso de las páginas web corporativas principalmente como herramientas comerciales, tanto en el caso de las grandes cadenas hoteleras como en el de los hoteles administrados de forma independiente. Este hecho se pone de manifiesto sobre todo en el gran número de empresas que aportan información a este colectivo en sus páginas web corporativas. En este sentido, se ha comprobado que los clientes son el grupo de interés mejor atendido a través de medios electrónicos, a veces con una distribución muy desigual con respecto a otros colectivos, como los proveedores o accionistas, que reciben información de RSC en un número significativamente menor de páginas web. Además, se ha descubierto que un elevado porcentaje de los hoteles muestran motores de reserva en línea como un medio para la venta de sus servicios a través de sus portales web.

En línea con este carácter comercial identificado en las páginas web, los autores también han encontrado que un gran volumen de empresas informan sobre temas de RSC que están directamente vinculados a la actividad principal de la empresa. En concreto, un gran número de empresas proporcionan información en términos de patrimonio natural y recursos culturales de los destinos turísticos, que son aspectos que afectan directamente a la capacidad de atracción de turistas, no sólo por parte de los destinos, sino también de los propios hoteles.

Estos últimos resultados, además, confirman el carácter contextual de la RSC en España, que debe ser reconocido y tenido en cuenta en la definición e implementación de la RSC por parte de las empresas en diversos sectores. Por ejemplo, los resultados de este estudio contradicen los hallazgos de Konrad et al. (2006) al analizar la RSC en un contexto multi-industria. Estos autores observaron que la dimensión económica del desarrollo sostenible era la más presente en la comunicación de la mayoría de empresas de diversos sectores ya que temas como el desempeño financiero y la competitividad a largo plazo eran los más frecuentemente comunicados por las empresas. Por lo tanto, las implicaciones de la RSC en la industria del turismo son muy diferentes a sus implicaciones en otros contextos económicos.

Los resultados relativos al papel de los proveedores en la comunicación de RSC de las empresas hoteleras también conducen a una conclusión relevante de este estudio. En este caso, es sorprendente corroborar la ausencia de una dimensión específica de información que recoja las implicaciones económicas de la relación comercial con proveedores. El análisis de las prácticas de RSC definidas en el sector en relación con dicho grupo de interés señala a que la comunicación corporativa está centrada más en las obligaciones impuestas por parte de las empresas hoteleras a sus proveedores que en las prácticas que realmente representan un beneficio mutuo para ambas partes. En este sentido, las categorías de información más frecuentemente identificadas en el estudio versan sobre el establecimiento de códigos de conducta, los procedimientos para la compra responsable, la estimulación de la responsabilidad de los proveedores en términos sociales y culturales y la estimación del impacto medioambiental de la cadena de suministro.

En términos de RSC, por lo tanto, los proveedores parecen a merced de la voluntad de las grandes cadenas y hoteles independientes, lo que en muchos casos puede perjudicar la buena voluntad de los proveedores para con sus clientes, así como 
puede perjudicar la cooperación empresarial para una aplicación más exitosa de los principios de la RSC en la industria turística. Este resultado, además, va en la línea de los previamente identificados en la literatura académica (p.ej., Dodds y Kuehnel, 2010).

Cabe destacar también algunas implicaciones de gestión interesantes derivadas de los resultados hallados en este trabajo. En primer lugar, las empresas hoteleras deben tratar de mejorar la organización y la presentación de su información de RSC en Internet ya que ahora ésta se encuentra dispersa a través de sus sitios web y, a veces, la información más pertinente en cada dimensión y para cada grupo de interés no se presenta de forma clara e integrada. La organización de la comunicación de RSC siguiendo la estructura teórica propuesta en este artículo podría ayudar a las empresas en esta tarea.

En segundo lugar, se ha demostrado también que las empresas hoteleras deberían valoran en mayor medida la información cultural de los destinos turísticos en los que se encuentran situados porque éste es un componente clave para el desarrollo sostenible de la industria turística.

En tercer lugar, las empresas se beneficiarían en gran medida si se replanteasen su relación comunicativa con algunos de sus grupos de interés, como los proveedores, los cuales no están siendo informados de los temas clave para ellos, es decir, las cuestiones puramente más económicas.

En último lugar, cabe destacar también la importancia de que los hoteles independientes, que representan el mayor porcentaje de empresas en el sector, aumenten su implicación con la RSC y comuniquen en mayor medida las prácticas llevadas a cabo en este ámbito. De no ser así, estas empresas pueden estar poniendo en peligro su imagen corporativa al no informar sobre una temática cada vez más relevante para la sociedad y los grupos de interés.

Finalmente, conviene aclarar que el estudio no está exento de limitaciones. En primer lugar, la investigación ha tenido una finalidad exploratoria y, por lo tanto, los autores no han aplicado pruebas estadísticas suficientes para demostrar la fiabilidad y validez del marco teórico presentado en este trabajo. El objetivo de la investigación ha sido el de presentar un enfoque integrador para el estudio de la RSC en el sector hotelero, desde una perspectiva claramente normativa, y aplicándolo con fines descriptivos en el sector hotelero. Una vez que el enfoque ha quedado descrito y explicado, futuras investigaciones deberán estar enfocadas a diseñar escalas de medición, fiables y completas, que ayuden a investigadores y profesionales del sector a identificar mejores prácticas de comunicación de RSC desde el enfoque del presente trabajo.

En esta línea, también es evidente que los autores han evaluado la comunicación de RSC únicamente teniendo en cuenta la cantidad de información proporcionada en Internet por parte de los hoteles. No obstante, la calidad de la información proporcionada por las empresas también es relevante en el estudio de la comunicación de RSC (Piechocki, 2004). Futuras líneas de investigación deben ofrecer formas más sofisticadas para evaluar la comunicación de RSC, teniendo también en cuenta la calidad de la información.

En tercer lugar, los autores también han encontrado problemas de comparabilidad en la información de RSC proporcionada por las empresas hoteleras del estudio. Este problema también ha surgido en estudios previos, como el de Bonilla y Avilés (2008). En este sentido, aunque la mayoría de los hoteles informan sobre las mismas cuestiones 
económicas, sociales, ambientales, culturales y de grupos de interés, la información no es del todo comparable porque no hay suficiente información disponible acerca de cómo las empresas han medido los datos reportados. Incluso cuando los hoteles utilizan los mismos indicadores, no siempre usan las mismas unidades de información, como en el caso de la presentación de los resultados relativos al desperdicio de recursos y el reciclaje, que en algunos casos se mide en término de volumen/peso mientras que, en otras ocasiones, se reporta en unidades monetarias. Las investigaciones futuras deberían tratar de controlar esta especificidad de la comunicación de RSC.

Por último, el marco conceptual presentado en este trabajo se ha aplicado a un contexto de investigación limitado (empresas hoteleras en España). Por lo tanto, su idoneidad para entender la comunicación de RSC en otras regiones no ha sido probada aún. Nuevos estudios deben probar el modelo conceptual en diferentes contextos de investigación con el fin de corroborar y ampliar las conclusiones extraídas de este trabajo.

\section{BIBLIOGRAFÍA}

AGARWAL, S. (2002): «Restructuring Seaside Tourism: The Resort Lifecycle», Annals of Tourism Research, vol 29, $\mathrm{n}^{\circ}$ 1, pp. 25-55.

AYUSO, S. y FULLANA, P. (2002): Sustainable Tourism. Barcelona, Rubes.

BAKEMAN R. y GOTTMAN, J.M. (1986): Observing Interaction: An Introduction to Sequential Analysis. Cambridge, Cambridge University Press.

BOHDANOWICZ, P. y ZIENTARA, P. (2008): «Hotel Companies' Contribution to Improving the Quality of Life of Local Communities and the Well-being of their Employees», Tourism and Hospitality Research, vol. 9, n 2, pp. 147-158.

BONILLA, M.J. y AVILÉS, C. (2008): «Analysis of Environmental Statements Issued by EMAS-Certified Spanish Hotels», Cornell Hospitality Quarterly, vol 49, no 4, pp. 381-394.

BRAVO, R., MATUTE, J. y PINA, J.M. (2012): «Corporate Social Responsibility as a Vehicle to Reveal the Corporate Identity: A Study Focused on the Websites of Spanish Financial Entities», Journal of Business Ethics, vol. 107, n 2, pp. 129-146.

CLARKSON, M.B.E. (1995): «A Stakeholder Framework for Analyzing and Evaluating Corporate Social Performance», Academy Management Review, vol. 20, pp. 92-117.

DE GROSBOIS, D. (2012): «Corporate Social Responsibility Reporting by the Global Hotel Industry: Commitment, Initiatives and Performance», International Journal of Hospitality Management, vol. 31, pp. 896-905.

DODDS, R. y KUEHNEL, J. (2010): «CSR among Canadian Mass Tour Operators: Good Awareness but Little Action», International Journal of Contemporary Hospitality Management, vol. 22, n 2, pp. 221-244.

ESROCK, S.L. y LEICHTY, G.B. (1998): «Social Responsibility and Corporate Web Pages: Self-presentation or Agenda-setting? », Public Relations Review, vol. 4, $\mathrm{n}^{\circ} 3$, pp. 305-319.

EXCELTUR (2003): Acción Social en la Empresa Turística. Alianza para la Excelencia Turística, Madrid. 
FLOREK, M., INSCH, A. y GNOTH, J. (2006): «Papers City Council Websites as a Means of Place Brand Identity Communication», Place Branding, vol. 2, $\mathrm{n}^{\circ}$ 4, pp. 276-296.

FREEMAN, R.E. (1984): Strategic Management: A Stakeholder Approach. Boston, Pittman.

FONT, X., WALMSLEY, A., COGOTTI, S., MCCOMBES, L. y HÄUSLER, N. (2012): «Corporate Social Responsibility: The Disclosure-performance Gap», Tourism Management, vol. 33, pp. 1.544-1.553.

GRAM, M. (2007): «Children as Co-Decision Makers in the Family? The Case of Family Holidays», Young Consumers, vol. 8, n 1, pp. 19-28.

GRÖSCHL, S. (2011): «Diversity Management Strategies of Global Hotel Groups: A Corporate Web Site Based Exploration», International Journal of Contemporary Hospitality Management, vol. 23, $\mathrm{n}^{\circ}$ 2, pp. 224-240.

HOLCOMB, J.L., UPCHURCH, R.S. y OKUMUS, F. (2007): «Corporate Social Responsibility: What are Top Hotel Companies Reporting?», International Journal of Contemporary Hospitality Management, vol., n ${ }^{\circ}$ 6, pp. 461-476.

JONES, P., COMFORT, D. e HILLIER, D. (2006): «Reporting and Reflecting on Corporate Social Responsibility in the Hospitality Industry: A Case Study of Pub Operators in the UK», International Journal of Contemporary Hospitality Management, vol. 18, $\mathrm{n}^{\circ}$ 4, pp. 329-340.

KONRAD, A., STEURER, R., LANGER, M.E. y MARTINUZZI, A. (2006): «Empirical Findings on Business-Society Relations in Europe», Journal of Business Ethics, vol. 63, pp. 89-105.

MARTÍNEZ, R.P., PÉREZ, A. y RODRÍGUEZ DEL BOSQUE, I. (2013): «Measuring Corporate Social Responsibility in Tourism: Development and Validation of an Efficient Measurement Scale in the Hospitality Industry», Journal of Travel \& Tourism Marketing, vol. 30, pp. 365-385.

MERWE, M.C.S. y WÖCKE, A. (2007): «An Investigation into Responsible Tourism Practices in the South African Hotel Industry», South African Journal of Business Management, vol. 38, $\mathrm{n}^{\mathrm{o}} 1-12$.

MICHELON, G. (2011): «Sustainability Disclosure and Reputation: A Comparative Study», Corporate Reputation Review, vol. 14, n 2, pp. 79-96.

MORSING, M. y SCHULTZ, M. (2006): «Corporate Social Responsibility Communication: Stakeholder Information, Response and Involvement Strategies», Business Ethics: A European Review, vol. 15, n 1, pp. 323-338.

NEÜENDORF, K.A. (2002): The Content Analysis Guidebook. Thousand Oaks, Sage Publications.

PERRINI, F., POGUTZ, S. y TENCATI, A. (2006): «Corporate Social Responsibility in Italy: State of the Art», Journal of Business Strategies, vol. 23, n 1, pp. 65-91.

PIECHOCKI, R. (2004): «Industry Survey: Transparency of Annual Sustainability Reports», Corporate Reputation Review, vol. 7, n 2, pp. 107-123.

POMERING, A. y DOLNICAR, S. (2009): «Assessing the Prerequisite of Successful CSR Implementation: Are Consumers Aware of CSR Initiatives?», Journal of Business Ethics, vol. 85, pp. 285-301. 
ROWLEY, J. (2009): «Online Branding Strategies of UK Fashion Retailers», Internet Research, vol. 19, n 3, pp. 348-369.

WILLIAMS, S.J. y ADAMS, C.A. (2013): «Moral Accounting? Employee Disclosures from a Stakeholder Accountability Perspective», Accounting, Auditing and Accountability Journal, vol. 26, no 3, pp. 449-495. 